\title{
Selecting appropriate ERP software using integrated fuzzy linguistic preference relations - fuzzy TOPSIS method
}

\author{
Süleyman Çakır \\ Department of Business Administration, Recep Tayyip Erdoğan University, \\ 53100, Rize, Turkey \\ E-mail: suleyman.cakir@erdogan.edu.tr
}

Received 1 October 2014

Accepted 3 February 2016

\begin{abstract}
Due to the high uncertainty of business environment, the complexity and diversity of enterprise resource planning (ERP) projects and conflicting assessment criteria, appropriate ERP software selection can be viewed as a multi-criteria decisionmaking (MCDM) problem. Among the MCDM methods, extent analysis method (EAM) has been employed in many applications due to its computational simplicity. However, in EAM, inconsistency rises as the numbers of criteria or alternatives increase. In order to address this inconsistency problem, fuzzy linguistic preference relations (FLPR) has been proposed to obtain consistent solutions in the decision-making processes. This study proposes a robust ERP selection framework where the weights of the evaluation criteria are computed through FLPR and the ranking of the alternative ERP systems is obtained via Fuzzy TOPSIS. A real life application has been executed in a Turkish wholesale food company.
\end{abstract}

Keywords: Enterprise resource planning (ERP), Fuzzy linguistic preference relations (FLPR), Fuzzy TOPSIS, Multiple criteria analysis.

\section{Introduction}

Enterprise resource planning (ERP) is a combined enterprise information system integrating crucial business functions such as production planning, purchase, inventory control, sales, finance, and human resource $^{1}$. The main aim of a typical ERP system is fusing the best practices and processes of multiple organizations located in different geographical places with varying requirements. Facing severe market competition, companies seek to select optimum ERP system in order to gain competitive advantage, reduce total cost, maximize return on investment, shorten lead times and be more responsive to customer requirements ${ }^{2}$. Implementing ERP systems properly is a challenging process because of its complexity, high cost and adaptation risks. While considerable number of ERP projects have attained worthy improvements in both tangible and intangible areas, many organizations have failed to draw sufficient benefits from their ERP systems ${ }^{3}$.

A successful ERP project includes selecting an ERP software system and vendor, executing this system, managing business processes change and checking the practicality of the system ${ }^{4}$. Firms have spent billions $\$$ and utilized huge amounts of working-hours for introducing elaborate ERP software systems. ${ }^{5}$. The large capital investment required to install ERP systems implies to be very picky in the ERP system selection decision process. Given the far-reaching effects on the 
organization's use of the ERP system and its subsequent performance outcome, it is obvious that the way implementation is deployed can facilitate or impede the ultimate performance outcome ${ }^{6}$. Furthermore, there is an expanding unanimity among ERP system implementers that selecting an inappropriate system is one of the main culprits for ERP implementation failure7.

Due to the high uncertainty of business environment, the complexity and diversity of ERP projects and conflicting assessment criteria, appropriate ERP software selection among alternatives can be viewed as a multi-criteria decision making (MCDM) problem. MCDM methods refer to obtain the optimal alternative among feasible alternatives in the presence of multiple, usually conflicting evaluation criteria.

Because of its computational simplicity and inherent capability to handle tangible and intangible criteria, Analytical Hierarchy Process ${ }^{8}$ (AHP) is one of the most utilized MCDM method in selecting ERP systems and other decision-making problems. The foremost characteristic of AHP is combining knowledge, experience, individual opinions and foresights through pairwise comparisons. Pairwise comparisons provide a flexible and realistic way to model real-life problems. However, due to the complexity and uncertainty of realworld problems, decision-makers are often unable to assign exact judgments to the decision criteria. In addition, the human judgments made in the decision process involve inherent subjectivity and are often infeasible and inconsistent. Therefore, it is more suitable to introduce data using fuzzy numbers instead of crisp numbers ${ }^{9}$. Because of the above-mentioned pitfalls, alternative methods for improving classical AHP have been developed by several authors by employing fuzzy set theory.

Fuzzy AHP is an important extension of the conventional AHP method which was first introduced by Laarhoven and Pedrycz ${ }^{10}$. They suggested a fuzzy logarithmic least squares method (LLSM) to attain triangular fuzzy weights from a triangular fuzzy comparison matrix. Buckley ${ }^{11}$ extended the AHP using the geometric mean method to calculate fuzzy weights. Chang $^{12}$ developed an extent analysis method (EAM) which derives crisp weights for fuzzy comparison matrices. EAM has been used in many applications since its computational simplicity. In EAM, inconsistency escalates as the numbers of criteria or alternatives increase. Decision-makers often have difficulty in providing a consistent pairwise comparison among conflicting criteria. In an effort to address the mentioned problems, Herrera-Viedma et al. ${ }^{13}$ proposed consistent fuzzy preference relations to achieve consistent solutions in the decision-making processes. Furthermore, while performing the AHP requires $\frac{n *(n-1)}{2}$ pairwise comparisons consistent fuzzy preference relations entails only (n-1) comparisons ${ }^{14}$. Wang and $\mathrm{Chen}^{15-17}$ and $\mathrm{Chen}^{18}$ presented a method using fuzzy linguistic variables instead of crisp values to form fuzzy linguistic preference relations (FLPR) matrices based on consistent fuzzy preference relations. Their method ensures consistency and only necessitates (n-1) judgments from a set of $\mathrm{n}$ elements.

Technique for order preference by similarity to ideal solution (TOPSIS) introduced by Hwang and Yoon ${ }^{19}$ is another highly preferred MCDM technique in the literature. In the TOPSIS theory, crisp values are used for weights and performance ratings of the criteria. Chen $^{20}$ extended the TOPSIS method to the fuzzy environment and offered a vertex method to calculate the distance between two triangular fuzzy numbers (TFNs).

This study proposes a novel ERP selection framework where the weights of the evaluation criteria are computed through FLPR and the ranking of the alternative ERP systems is attained via Fuzzy TOPSIS proposed by $\mathrm{Chen}^{20}$. By applying the proposed hybrid method it is aimed to exploit the advantageous characteristics of both methods. A real life application is executed in a Turkish wholesale food company.

The remainder of the paper is organized as follows. Section 2 presents the literature regarding ERP software/vendor selection. Section 3 covers the procedures of FLPR and Fuzzy TOPSIS methods. Section 3 displays the real case application of the proposed combined model. Conclusions are provided in the final section.

\section{Literature review}

In recent decades, ERP or other information systems (IS) selection problems have been addressed by a number of methods including scoring, ranking, mathematical optimization and multi-criteria decision 
analysis. Due to having over simplicity, the scoring method $^{21-22}$ is a highly demanding technique, yet it is unable to reflect decision makers' opinions. The ranking approach $^{23}$ also has the same drawbacks with the scoring method ${ }^{2}$. Various methods such as goal programming, 0-1 programming and nonlinear programming have also been proposed for the optimal IS selection. Santhanam and Kyparisis ${ }^{24}$ run nonlinear zero-one goal programming (ZOGP) model for a suitable IS project selection between 14 alternatives for a large fast food company. The model used in their paper considered inter-dependencies among projects in the IS selection process. The model, then, is converted to a linear mixed integer programming model via a linearization technique. Lee and $\mathrm{Kim}^{25}$ suggested an enhanced IS project selection methodology which covers inter-dependencies among evaluation criteria and candidate projects using analytic network process (ANP) within a ZOGP model. Lall and Teyarachakul ${ }^{26}$ used Data Envelopment Analysis (DEA) to selecting the best ERP software between alternatives by taking into consideration of two qualitative and two quantitative criteria. Karsak and Özoğul ${ }^{7}$ developed a novel decision framework for ERP software selection based on combined quality function deployment (QFD), fuzzy linear regression and ZOGP methods. The proposed framework enables both company demands and ERP system characteristics to be considered. Badri et al. ${ }^{27}$ offered using a $0-1$ goal programming model to select an IS project and compared the results with the ranking method. The developed model is compatible with group decision-making and overcomes the infeasibility pitfalls of the ranking method. However, the viability of the above mentioned methods is declined by ascending complexity as the number of IS alternatives and evaluation criteria get increased or some attributes are not easily measurable ${ }^{27}$.

Teltumbde ${ }^{28}$ proposed a framework based on the nominal group technique and AHP so as to evaluate three ERP projects by taking account of ten decision criteria. The study focused on the elaboration of some common criteria for ERP evaluation. Wei and Wang ${ }^{29}$ proposed using integrated fuzzy average method and fuzzy integral ranking for choosing an ERP project in which a hierarchical attribute structure including project, software and vendor factors were provided. The final decision is determined by the highest total integral value. Wei et al. ${ }^{2}$ presented a comprehensive framework based on the AHP method to choosing the suitable ERP system. The authors established an objective hierarchy where appropriate attributes were elicited to supply inclusive guidance for ERP system evaluation. Yazgan et al. ${ }^{1}$ suggested a novel approach including an artificial neural network (ANN) model designed and trained with using ANP results so as to calculate ERP software priority. In the suggested model, once ANP and ANN models are constructed ERP software selection can be made easily by the opinions of one single expert. Karaaslan and Gündoğar ${ }^{30}$ utilized AHP to select the best ERP alternative between two alternatives for a marble machine manufacturer. The proposed framework can systematically establish the objectives of ERP selection to support the business goals and strategies of a company. Besides, it can specify the suitable attributes and set up a consistent evaluation standard for facilitating a group decision process. Lin et al. ${ }^{31}$ introduced a combined framework including ANP, TOPSIS and linear programming (LP) to develop a realtime purchasing environment for an electronics company through an ERP system. ANP and TOPSIS were used to calculate the weight and give suppliers a ranking, then, LP effectively allocates order quantity to each vendor. Gürbüz et al. $^{32}$ proposed a novel framework integrating ANP, Choquet integral (CI) and Measuring attractiveness by a categorical based evaluation technique (MACBETH) in order to select the suitable ERP project among four alternatives. ANP produces the priorities of alternatives with respect to the inter-dependent evaluation criteria. The conjunctive or disjunctive behaviors between criteria are specified using MACBETH and CI. Other studies addressing decision support systems selection problem includes the works of Zhang et al. ${ }^{33}$ and Liu et al. ${ }^{34}$.

\section{Methodology}

This paper performs FLPR to fuzzify hierarchical analysis by using fuzzy numbers for the pairwise comparisons and thereby computes the fuzzy preference weights. In this section, three different MCDM methods are presented. The first one is consistent fuzzy preference relations developed by Herrera-Viedma et $\mathrm{al}^{13}$. The second one is FLPR proposed by Wang and Chen ${ }^{15-17}$ and $\mathrm{Chen}^{18}$. Finally, fuzzy TOPSIS introduced by $\mathrm{Chen}^{20}$ is summarized. 


\subsection{Fuzzy Sets}

Fuzzy sets theory has firstly been introduced by Zadeh ${ }^{35}$ as an extension of classical (crisp) sets. Contrary to crisp sets, a fuzzy set can be constituted via assigning a membership value to each element in the interval of $[0,1]$. Membership values represent the degree to which an element belongs to a fuzzy set. A fuzzy set is denoted by its membership function $\mu_{A}(x)$ as:

$$
\mu_{A}(x): X \rightarrow[0,1]
$$

\subsubsection{Fuzzy Numbers}

A fuzzy number is described on the universe $\mathfrak{R}$ as a convex and normalized fuzzy set ${ }^{36}$. TFNs are the most commonly used form of fuzzy sets since having intuitive membership function. A TFN $\widetilde{A}$ is defined on $\mathfrak{R}$ with the membership function $\mu_{A}(x)$ by:

$$
\mu_{A}(x)=\left\{\begin{array}{lc}
(x-l) /(m-l), & l \leq m \leq x \\
(u-x) /(u-m), & m \leq x \leq u \\
0, & \text { otherwise }
\end{array}\right.
$$

Herein, $l, m$ and $u$ denote the lower, modal value and upper bounds of the fuzzy number $\tilde{A}$, respectively. Then, a TFN can be represented by $\widetilde{A}=(l, m, u)$.

\subsection{Consistent fuzzy preference relations}

Being a useful tool in modeling decision processes, preference relations are the most common representation of information used in decision-making problems. In a preference relation an expert or decisionmaker assigns values to a set of criteria and a set of alternatives that reflects some degree of preference of the first alternative over the second one. There exist two most common preference relations used in the decisionmaking literature: (i) multiplicative preference relations, and (ii) fuzzy preference relations ${ }^{17}$.

(i) Multiplicative preference relations ${ }^{36}$ :

A multiplicative preference relation $A$ on a set of alternatives $X$ is denoted by $a$ matrix $A \subseteq X \times X, A=\left(a_{i j}\right)_{n x n}, a_{i j} \in[1 / 9,9]$ where $a_{i j}$ is the preference ratio of alternative $x_{i}$ to $x_{j}$. Saaty suggests measuring $a_{i j}$ using a ratio scale, namely, the 1-9 scale where $a_{i j}=1$ indicates indifference between $x_{i}$ and $\mathrm{x}_{\mathrm{j}}$; $a_{i j}=9$ indicates that $x_{i}$ is absolutely preferred to $x_{j}$. In this case, the preference relation $A$ is usually assumed to be multiplicative reciprocal, i.e.

$a_{i j} \cdot a_{j i}=1 \quad \forall i, j \in\{1, \ldots, n\}$

(ii) Fuzzy Preference Relations ${ }^{37-39}$

A fuzzy preference relation $P$ on a set of alternatives $A$ is a fuzzy set on the product set $A \times A$ with membership function $\mu_{p}: A \times A \rightarrow[0,1]$ The preference relation is denoted by $n \times n$ matrix $P=\left(p_{i j}\right), \quad$ where $p_{i j}=\mu_{p}\left(a_{i}, a_{j}\right) \forall i, j \in\{1, \ldots, n\} \quad$ denotes the reference ratio of alternative $a_{i}$ to that of $a_{j}$. When $p_{i j}=1 / 2$, it implies indifference between $a_{i}$ and $a_{j} ; p_{i j}=1$ indicates that $a_{i}$ is absolutely superior to $a_{i}$ and $p_{i j}>1 / 2$ refers to $a_{i}$ is better than and preferred to $a_{i}$. The preference matrix $P$ is assumed to be an additive reciprocal given by $p_{i j}+p_{j i}=1, \forall i, j \in\{1, \ldots, n\}$.

Fedrizzi $^{40}$ proved that by means of function $g$, a "multiplicative" formulation of a problem can be transformed into an additive one. For a set of alternatives $X=\left\{x_{1}, . ., x_{n}\right\}$ associated with a reciprocal multiplicative preference relation $A=a_{i j}$ and $a_{j} \in[1 / 9,9]$, transformation function $g$ can be used as in Eq. (2) to find the corresponding reciprocal additive fuzzy preference relation $P=\left(p_{i j}\right)$ and $p_{i j} \in[0,1]$.

$p_{i j}=g\left(a_{i j}\right)=1 / 2 \cdot\left(1+\log _{9} a_{i j}\right)$

Herein, $\log _{9} a_{i j}$ regarded since $a_{i j}$ is between $1 / 9$ and 9 . In case $a_{i j}$ is between $1 / 7$ and 7 , then $\log _{7} a_{i j}$ is utilized. Herrera-Viedma et al. ${ }^{13}$ proposed that for a reciprocal additive fuzzy preference relation $P=\left(p_{i j}\right)$, the following statements are equivalent:

$$
\begin{aligned}
& p_{i j}+p_{j k}+p_{k i}=3 / 2 \forall i \prec j \prec k, \\
& p_{i(i+1)}+p_{(i+1)(i+2)}+\ldots \ldots+p_{(j i)}+p_{j i}=\frac{j-i+1}{2}=3 / 2 \square i \prec j,
\end{aligned}
$$

From the above Eq. (4), it can be deduced that

$p_{j i}=\frac{j-i+1}{2}-p_{i(i+1)}-p_{(i+1)(i+2)}-\ldots-p_{(j-1) j}$

and based on the additive reciprocal,

$$
p_{i j}+p_{j i}=1 \forall i, j \in\{1, \ldots, n\}
$$

The procedure of fuzzy preference relations is outlined as below.

Step 1. Compute the set of preference values $B$ as

$$
B=\left\{p_{i j}, i \prec j \wedge p_{i j} \quad\left\{p_{12}, p_{23}, \ldots, p_{n}{ }_{1 n}\right\}\right\}
$$


Step 2. Comprise $P$

$$
P=\left\{p_{12}, p_{23}, \ldots, p_{n-1 n}\right\} \cup \mathrm{B} \cup\left\{1-p_{12}, 1-p_{23}, \ldots, 1 \quad p_{n-1 n}\right\} \cup \neg B .
$$

Step 3. The consistent fuzzy preference relation $P$ is achieved as $P^{\prime}=f:[-a, 1+a] \rightarrow[0,1]$

$$
f(x)=\frac{x+a}{1+2 a}
$$

The rationale of this method is that for $n$ attributes $X=\left\{x_{1}, \ldots, x_{n} \quad n \geq 2\right\}$ the pairwise preference relation data $\left\{p_{12}, p_{23}, \cdots, p_{n-1 n}\right\}$ can be extracted by comparing only (n-1) attributes and constructing a consistent reciprocal fuzzy preference relations $P$. This method is akin to that for classical AHP characteristics, which is a transitivity property satisfied by preference relations.

\subsection{Fuzzy linguistic preference relations (FLPR)}

Wang and $C h e n^{15-17}$ proposed a method using fuzzy linguistic assessments variables to constitute FLPR matrices based on consistent fuzzy preference relations. The FLPR has been acknowledged to improve the consistency of fuzzy AHP. The fuzzy linguistic assessments variables are given by

$\widetilde{P}=\left(\widetilde{p}_{i j}\right)=\left(p_{i j}^{L}, p_{i j}^{M}, p_{i j}^{R}\right)$ where $p_{i j}^{L}$ and $p_{i j}^{R}$ denote respectively, the lower and upper bounds of the fuzzy number $\widetilde{P}$ and $p_{i j}^{M}$ represents the median value. Some essential propositions are presented below ${ }^{15-18}$.

Proposition 3.3.1. Given a set of alternatives $X=\left\{x_{1}, \ldots, x_{n}\right\}$ associated with a fuzzy reciprocal multiplicative preference relation $\widetilde{A}_{i j}=\left(\widetilde{a}_{i j}\right)$ for $\widetilde{a}_{i} \in[1 / 9,9]$, and the corresponding fuzzy reciprocal linguistic preference relation $\widetilde{P}_{i j}=g\left(\widetilde{p}_{i j}\right)=1 / 2\left(1+\log _{9} \widetilde{a}_{i j}\right)$ with $\widetilde{p}_{i j} \in[0,1]$, hence, the following statements are equivalent:

(i) $p_{i j}^{L}+p_{j i}^{R}=1 \forall i, j \in\{1, \ldots, n\}$,

(ii) $p_{i j}^{M}+p_{j i}^{M}=1 \forall i, j \in\{1, \ldots, n\}$,

(iii) $p_{i j}^{R}+p_{j i}^{L}=1 \forall i, j \in\{1, \ldots, n\}$.

Proposition 3.3.2. For a consistent reciprocal fuzzy linguistic preference relation $\widetilde{P}_{i j}=\left(\widetilde{p}_{i j}\right)=\left(p_{i j}^{L}, p_{i j}^{M}, p_{i j}^{R}\right)$, the following statements are equivalent:
(a) $p_{i j}^{L}+p_{j k}^{L}+p_{k i}^{R}=3 / 2 \quad \forall i \prec j \prec k$,
(b) $p_{i j}^{M}+p_{j k}^{M}+p_{k i}^{M}=3 / 2 \forall i \prec j \prec k$,
(c) $p_{i j}^{R}+p_{j k}^{L}+p_{k i}^{L}=3 / 2 \forall i \prec j \prec k$,

(d) $p_{i(i+1)}^{L}+p_{(i+1)(i+2)}^{L}+\ldots+p_{(j-1) j}^{L}+p_{j i}^{R}=\frac{j-i+1}{2} \forall i \prec j$,

(e) $p_{i(i+1)}^{M}+p_{(i+1)(i+2)}^{M}+\ldots+p_{(j-1) j}^{M}+p_{j i}^{M}=\frac{j-i+1}{2} \forall i \prec j$,

(f) $p_{i(i+1)}^{R}+p_{(i+1)(i+2)}^{R}+\ldots+p_{(j-1) j}^{R}+p_{j i}^{L}=\frac{j-i+1}{2} \forall i \prec j$.

Notably, if the values of the acquired matrix $\widetilde{P}$ with entries $\widetilde{p}_{i j}$ in the interval $[-c, 1+c](c>0)$ are not in the interval $[0,1]$, in order to preserve reciprocity and additive consistency $f:[-c, 1+c] \rightarrow[0,1]$, the achieved fuzzy numbers would need to be converted via the following transformation function:

$$
f\left(x^{L}\right)=\frac{x^{L}+c}{1+2 c}, f\left(x^{M}\right)=\frac{x^{M}+c}{1+2 c}, f\left(x^{R}\right)=\frac{x^{R}+c}{1+2 c}
$$

The algorithm of FLPR is outlined below.

Step 1. Let $X=\left\{x_{1}, \ldots, x_{n}\right\}$ be a set of alternatives for a decision-making problem. An incomplete consistent FLPR $\quad \overline{\widetilde{P}}=\left(\overline{\widetilde{p}}_{i j}\right)_{n x n} \quad$ with only $(n-1) \quad$ judgments $\left\{p_{12}, p_{23}, \ldots, p_{n-1 n}\right\}$ is formed through comparing each pair of alternatives.

Step 2. The known elements in $\widetilde{P}$ and Propositions 3.3.1 and 3.3.2 are exploited together so as to find out all unknown elements in $\widetilde{P}$. Thereby, the corresponding complete FLPR are attained.

Step 3. Executing the linguistic averaging operator

$$
\widetilde{A}_{i}=\frac{\sum_{j=1}^{n} \overline{\widetilde{p}}_{i j}}{n} \text { for all } i
$$

the averaged $\widetilde{A}_{i}$ of the ith criterion (alternative) over all other criteria (alternatives) is calculated. The weight of $i$ is computed as,

$$
\widetilde{W}_{i}=\widetilde{A}_{i} / \sum_{i=1}^{n} \tilde{A}_{i}
$$

Step 4. Group integration and defuzzification The weight values of alternatives are denoted as fuzzy numbers, hence, defuzzification of fuzzy numbers is required for ranking alternatives. In this study, centroidof-area (COA) method is conveyed to ranking the fuzzy weights. The crisp value of the fuzzy weight of the $i$ th alternative denoted as a TFN $(l, m, u)$ is computed via the following formula.

$$
W_{i}=C O A\left(\widetilde{W}_{i}\right)=\frac{l+2 m+u}{4}
$$


Step 5. The priority of the alternatives is attained according to their ranking values extracted from Eq. (13).

\subsection{Fuzzy TOPSIS}

The underlying rationale of the TOPSIS method is to select an alternative which has the shortest distance from the positive-ideal solution and the longest distance from the negative-ideal solution. In the TOPSIS theory, crisp values are used for representing weights and performance ratings of the criteria. This method was extended to a fuzzy environment by Chen and Hwang ${ }^{19}$ to handle with uncertainties in decision making problems. Triantaphyllou and $\mathrm{Lin}^{41}$ introduced a fuzzy TOPSIS method based on fuzzy arithmetic operations, which computes a fuzzy relative closeness for each alternative. Chen ${ }^{20}$ extended the TOPSIS to the fuzzy environment and proposed a vertex method to calculate the distance between two TFNs. Chu and $\mathrm{Lin}^{42}$ proposed a fuzzy TOPSIS method for robot selection. Jahanshahloo et al. ${ }^{43}$ extended the TOPSIS method to decision-making problems with fuzzy data. In their paper, the rating of each alternative and the weight of each criterion are expressed in TFNs and the normalized fuzzy numbers are calculated by using the concept of $\alpha$ cuts. Krohling and Campanharo ${ }^{44}$ proposed a fuzzy TOPSIS for group decision making, which is applied to evaluate the ratings of response alternatives to a simulated oil spill. Paksoy et al. ${ }^{45}$ developed the organization strategy of distribution channel management using fuzzy AHP and hierarchical fuzzy TOPSIS for an edible-vegetable oils manufacturer firm operating in Turkey.

Table 1.Linguistic variables for importance weight of each criterion.

\begin{tabular}{ll}
\hline Linguistic variables & Triangular fuzzy numbers \\
\hline Very low (VL) & $(0,0,0.1)$ \\
Low (L) & $(0,0.1,0.3)$ \\
Medium low (ML) & $(0.1,0.3,0.5)$ \\
Medium (M) & $(0.3,0.5,0.7)$ \\
Medium high (MH) & $(0.5,0.7,0.9)$ \\
High (H) & $(0.7,0.9,1.0)$ \\
Very high (VH) & $(0.9,1.0,1.0)$ \\
\hline
\end{tabular}

Table 2. Linguistic variables for ratings

\begin{tabular}{ll}
\hline Linguistic variables & Triangular fuzzy numbers \\
\hline Very poor (VP) & $(0,0,1)$ \\
Poor (P) & $(0,1,3)$ \\
Medium poor (MP) & $(1,3,5)$ \\
Fair (F) & $(3,5,7)$ \\
Medium good (MG) & $(5,7,9)$ \\
Good (G) & $(7,9,10)$ \\
Very good (VG) & $(9,10,10)$ \\
\hline
\end{tabular}

The procedure of fuzzy TOPSIS is outlined as below $^{20,46}$.

Step 1. Initially a committee of involving $K$ decision makers is established. Fuzzy rating of each decision maker $D_{k}=(k=1, . ., K)$ can be denoted as a TFN $\widetilde{R}_{k}=(k=1, \ldots, K)$ with membership function $\mu_{\widetilde{R}_{k}}(x)$.

Step 2. The evaluation criteria are determined and then appropriate linguistic variables are chosen for evaluating criteria and alternatives using the linguistic variables demonstrated in Table 1 and Table 2, respectively.

Step 3. The weight of criteria are aggregated

Let the fuzzy ratings of all decision-makers are expressed as TFNs $\widetilde{R}_{k}=\left(a_{k}, b_{k, c_{k}}\right) k=1, \ldots, K$, then, the aggregated fuzzy rating can be shown as $\widetilde{R}=(a, b, c)$ $(k=1, . ., K)$. In here,

$$
a=\min _{k}\left\{a_{k}\right\}, b=\frac{1}{K} \sum_{k=1}^{K} b_{k}, c=\operatorname{mak}_{k}\left\{c_{k}\right\}
$$

Let the fuzzy rating and importance weight of the kth decision maker be $\widetilde{x}_{i j k}=\left(a_{i j k}, b_{i j k}, c_{i j k}\right)$ and

$$
\widetilde{w}_{i j k}=\left(w_{j k k}, w_{j k 2}, w_{j k 3}\right) \quad i=1, \ldots, m, \quad j=1, . ., n
$$

respectively, then the aggregated fuzzy ratings of alternatives with respect to each criterion can be computed as $\left(\tilde{x}_{i j}\right)=\left(a_{i j}, b_{i j}, c_{i j}\right)$.

Herein,

$$
a_{i j}=\min _{k}\left\{a_{i j k}\right\}, b=\frac{1}{K} \sum_{k=1}^{K} b_{i j k}, c=\operatorname{mak}_{k}\left\{c_{i j k}\right\}
$$

Accordingly, the aggregated fuzzy weights $\left(\widetilde{w}_{i j}\right)$ of each criterion are computed as,

$$
\widetilde{w}_{j}=\left(w_{j 1}, w_{j 2}, w_{j 3}\right)
$$

where

$$
w_{j l}=\min _{k}\left\{w_{j k 1}\right\}, w_{j 2}=\frac{1}{K} \sum_{k=1}^{K} w_{j k 2} w_{j 3}=\min _{k}\left\{w_{j k 3}\right\}
$$


Step 4. Then, the decision matrix $\widetilde{D}$ can be formed as:

$$
\widetilde{D}=\left[\begin{array}{cccc}
\widetilde{x}_{11} & \widetilde{x}_{12} & \ldots & \widetilde{x}_{I n} \\
\widetilde{x}_{11} & \widetilde{x}_{12} & \ldots & \widetilde{x}_{I n} \\
\vdots & \vdots & \ldots & \vdots \\
\widetilde{x}_{m 1} & \widetilde{x}_{m 2} & \ldots & \widetilde{x}_{m n}
\end{array}\right] \widetilde{W}=\left[\widetilde{w}_{1}, \widetilde{w}_{2}, \ldots, \widetilde{w}_{n}\right],
$$

where $\left(\tilde{x}_{i j}\right)=\left(a_{i j}, b_{i j}, c_{i j}\right)$ and $\tilde{w}_{j}=\left(w_{j l}, w_{j 2}, w_{j 3}\right)$ can be approximated by positive TFNs.

Step 5. The fuzzy decision matrix is normalized via linear scale transformation. The normalized fuzzy matrix $\widetilde{R}$ is denoted as,

$\widetilde{R}=\left[\widetilde{r}_{i j}\right]_{m x n}$ where $B$ and $C$ are the sets of benefit criteria and cost criteria, respectively, and

$$
\begin{aligned}
& \tilde{r}_{i j}=\left(\frac{a_{i j}}{c_{j}^{*}}, \frac{b_{i j}}{c_{j}^{*}}, \frac{c_{i j}}{c_{j}^{*}}\right), \quad j \in B, c_{j}^{*}=\max _{i} c_{i j} \quad j \in B \\
& \tilde{r}_{i j}=\left(\frac{a_{j}^{-}}{c_{i j}^{*}}, \frac{a_{j}^{-}}{b_{i j}^{*}}, \frac{a_{j}^{-}}{a_{i j}^{*}}\right), \quad j \in C, a_{j}^{-}=\min _{i} a_{i j} \quad j \in C
\end{aligned}
$$

Step 6. Considering the different importance of each criterion the weighted normalized decision matrix denoted by $\widetilde{V}$ is established as,

$$
\widetilde{V}=\left[\widetilde{v}_{i j}\right]_{m \times n} i=1, . ., m, j=1, . ., n
$$

where $\widetilde{v}_{i j}=\widetilde{r}_{i j}(.) \widetilde{w}_{i j}$

Step 7. According to the weighted normalized decision matrix, the elements of $\widetilde{v}_{i j}$ can be approximated by normalized TFNs. Therefore, the fuzzy positive ideal solution (FPIS, $A^{*}$ ) and fuzzy negative ideal solution (FNIS, $A^{*}$ ) can be expressed as,

$$
\begin{aligned}
& A^{*}=\left(\widetilde{v}_{1}^{*}, \widetilde{v}_{2}^{*}, . ., \widetilde{v}_{n}^{*}\right), \\
& A^{-}=\left(\widetilde{v}_{1}^{-}, \widetilde{v}_{2}^{-}, . ., \widetilde{v}_{n}^{-}\right),
\end{aligned}
$$

where $\tilde{v}_{j}^{*}=\max \left\{v_{i j 3}\right\}$ and $\tilde{v}_{j}^{-}=\min _{i}\left\{v_{i j 1}\right\} \quad(i=1, . ., m$; $j=1, . ., n)$

Step 8. The distance of each alternative from FPIS and FNIS are computed as,

$$
\begin{aligned}
& d_{i}^{*}=\sum_{j=1}^{n} d_{v}\left(\widetilde{v}_{i j}, \widetilde{v}_{j}^{*}\right), \quad i=1, \ldots, m \\
& d_{i}^{-}=\sum_{j=1}^{n} d_{v}\left(\widetilde{v}_{i j}, \widetilde{v}_{j}^{-}\right), \quad i=1, \ldots, m
\end{aligned}
$$

where $d_{v}(.,$.$) is the distance measurement between two$ fuzzy numbers.
Step 9. The closeness coefficient which represents the distances of each alternative to the FPIS and FNIS is calculated by the following formula.

$$
C C_{i}=\frac{d_{i}^{-}}{d_{i}^{*}+d_{i}^{-}} \quad i=1, \ldots, m
$$

The ranking order of alternatives would be elicited by descending order of $C C_{i}$ values. Obviously, an optimal alternative $A_{i}$ should be the closest one to FPIS and the farthest one to FNIS.

\section{The Proposed Evaluation Framework}

The proposed methodology aims to provide an analytical tool to select the most appropriate ERP software for a Turkish wholesale food company aiming to buy a new software system including online process following, purchasing processes, reporting services etc. The evaluation procedure of the application is comprised of seven steps as follows:

Step 1. Form an ERP project team

The first step of the proposed evaluation framework is to establish a project team involving managers, functional experts and senior representatives from user departments.

Step 2. Analyze company goals and demands and specify the ideal ERP system characteristics.

The project team clarifies the company goals and their expectations regarding the ideal ERP software.

Step 3. Compile the ERP vendors' evaluation criteria and technical features of the software.

The evaluation criteria of vendors and technical attributes of the ideal ERP software are determined by taking into account of related literature and teammembers' experiences. Then, these attributes will be used as the basis of the FLPR model.

Step 4. Constitute the hierarchical structure of the selection criteria.

The specified evaluation criteria in the prior step would be structured as a classical AHP hierarchy in the form of criteria, sub-criteria and alternatives.

Step 5. Gather opinions of team-members regarding the criteria, sub-criteria and alternatives in linguistic form via pairwise comparison. Here, a group decision-making approach, namely Delphi technique which comprises the opinions and judgments of all group while preempting their negative aspects such as social, personal 
and political conflicts, etc. ${ }^{47}$ is conducted. The project team evaluates the criteria and alternatives via pairwise comparison using linguistic variables presented in Table 3.

Step 6. Apply the FLPR procedure

Executing the FLPR procedure the relative importance ratings of the evaluation criteria are derived.

Step 7. Carry out fuzzy TOPSIS to ranking order the ERP software alternatives.

Fuzzy TOPSIS is applied in order to rank the ERP software alternatives.

Step 8. Discuss the results and select the best alternative.

A final evaluation of the attained results be carried out and then optimal ERP software is selected by project team. The flowchart of the selection process is displayed in Fig.1.

\subsection{Case study}

The proposed framework was applied to a Turkish wholesale food company operating in Blacksea Region of Turkey. The company's' sales network includes both some provinces of Turkey and the Republic of Azerbaijan, Georgia and Russia Federation. Operation managers of the company would like to make a decision on purchasing an ERP system for increasing the communication between the departments of the firm, lowering costs and stocks, strengthening supplier relationships and improving customer service. Initially, a project team of 5 consisting of a senior manager and two functional experts and two senior representatives of user departments was constituted. Then, the project team decided three main evaluation criteria and seventeen sub-criteria based on their experiences and studies in the literature. The evaluation criteria are grouped into three main categories as vendor factors, software factors and cost factors. Vendor factors (VF) contains four sub-criteria as technical-support level (TS), market position (MP), R\&D capability (RD) and good reputation (GR). System factors (SF) consists of functionality (FU), reliability (RE), ease of customization (EC), ease of implementation (EI) and efficiency (EF) while cost factors (CF) is comprised of price (PR), maintenance costs (MC), infrastructure cost (IC) and operating cost (OC). Accordingly, the hierarchy framework of the ERP selection process is shown in Fig. 2. In an effort to assign the criteria ratings, a comprehensive questionnaire concerning the related criteria for ERP software selection was prepared for the pairwise comparisons. Team-members evaluated the criteria and sub-criteria using the linguistic assessment variables listed in Table 3 .

Table 3. Fuzzy linguistic assessment variables and fuzzy numbers.

\begin{tabular}{ll}
\hline Linguistic Variables & Triangular Fuzzy Scale \\
\hline Very poor (VP) & $(0,0.0 .1)$ \\
Poor (P) & $(0,0.1,0.3)$ \\
Medium poor (MP) & $(0.1,0.3,0.5)$ \\
Medium (M) & $(0.3,0.5,0.7)$ \\
Medium good (MG) & $(0.5,0.7,0.9)$ \\
Good (G) & $(0.7,0.9,1)$ \\
Very good (VG) & $(0.9,1,1)$ \\
\hline
\end{tabular}

\subsubsection{FLPR Phase}

The pairwise comparison matrix of 3 main criteria with respect to goal is demonstrated in Table 4.

Table 4. Pairwise comparison of main criteria with respect to the goal.

\begin{tabular}{lccc}
\hline Goal & $\begin{array}{c}\text { Vendor } \\
\text { Factors }\end{array}$ & $\begin{array}{c}\text { System } \\
\text { Factors }\end{array}$ & $\begin{array}{c}\text { Cost } \\
\text { Factors }\end{array}$ \\
\hline Vendor & $\mathrm{X}$ & $\mathrm{P}$ & $\mathrm{X}$ \\
Factors & & & $\mathrm{G}$ \\
System & & $\mathrm{X}$ & $\mathrm{X}$ \\
Factors & & & $\mathrm{X}$ \\
Cost & & & \\
Factors & & & \\
\hline
\end{tabular}

As can be seen in Table 4, while 3 criteria were adopted only $2(n-1)$ comparison judgments $\left(p_{12}, p_{23}\right)$ were required to construct the fuzzy preference relation decision matrix. Herein, " $x$ " indicates the remaining $\widetilde{a}_{i j}^{k}$ calculated through Propositions 3.3.1 and 3.3.2. The whole calculation is as follows:

$$
\begin{aligned}
& p_{3 l}^{L}=1.5-p_{12}^{R}-p_{23}^{R}=1.5-0.3-1=0.2, \\
& p_{3 l}^{M}=1.5-p_{12}^{M}-p_{23}^{M}=1.5-0.1-0.9=0.5, \\
& p_{3 l}^{R}=1.5-p_{12}^{L}-p_{23}^{L}=1.5-0-0.7=0.8
\end{aligned}
$$


S. Çakır / Selecting appropriate ERP software using integrated FLPR

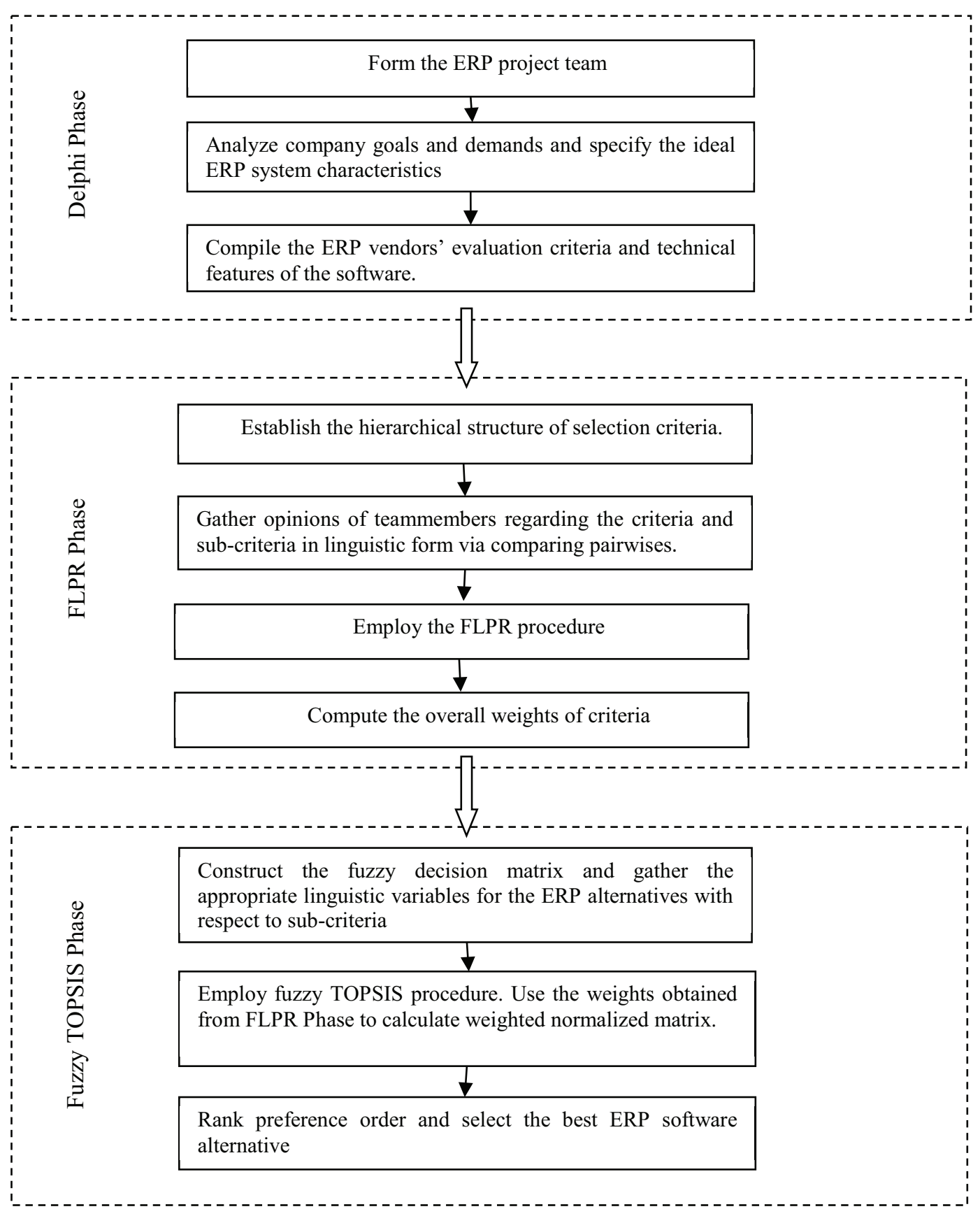

Fig. 1. The flowchart of the evaluation framework

Table 5 displays the complete consistent FLPR matrix related to main criteria.

Via Eq. (11) the average $\left(\widetilde{A}_{i}\right)$ for the main criteria is computed as:

$\mathrm{VF}=(0.23,0.37,0.53) \quad \mathrm{SF}=(0.63,0.77,0.83)$
$\mathrm{CF}=(0.23,0.37,0.53)$
Table 5. Complete FLPR matrix for main criteria.

\begin{tabular}{cccc}
\hline & VF & SF & CF \\
\hline VF & $(0.5,0.5,0.5)$ & $(0.0,0.1,0.3)$ & $(0.2,0.5,0.8)$ \\
SF & $(0.7,0.9,1.0)$ & $(0.5,0.5,0.5)$ & $(0.7,0.9,1.0)$ \\
CF & $(0.2,0.5,0.8)$ & $(0.0,0.1,0.3)$ & $(0.5,0.5,0.5)$ \\
\hline
\end{tabular}


S. Çakır / Selecting appropriate ERP software using integrated FLPR

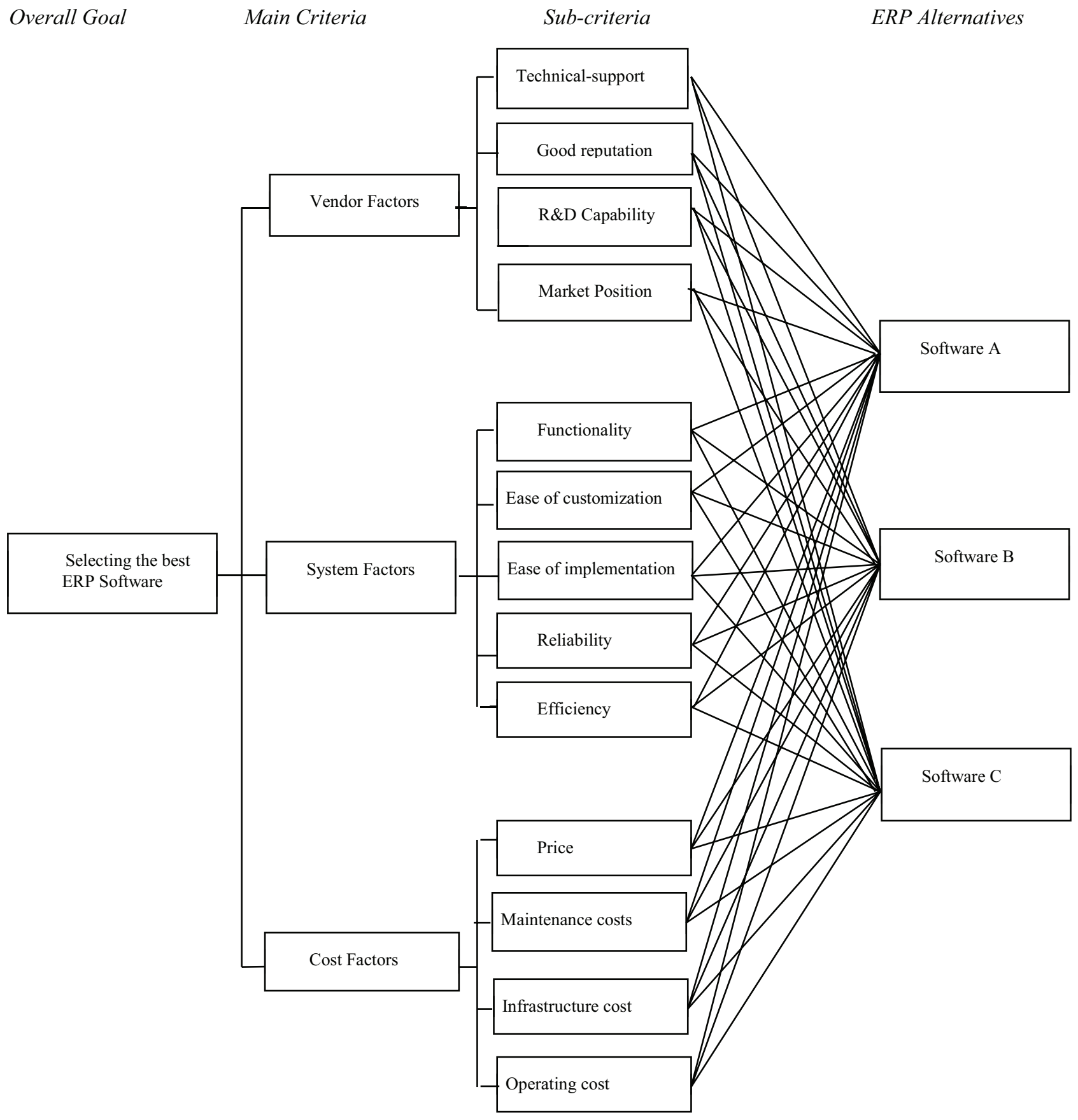

Fig. 2. The hierarchy of the proposed model 
The weight of each criterion is calculated using Eq. (12) as:

$$
\begin{aligned}
& \mathrm{VF}=(0.12,0.24,0.48) \quad \mathrm{SF}=(0.33,0.51,0.76) \\
& \mathrm{CF}=(0.12,0.24,0.48)
\end{aligned}
$$

The fuzzy weight values of criteria are represented in terms of fuzzy numbers. Using Eq. (13) a crisp value for each criterion is obtained as follows.

$$
\mathrm{VF}=(0.274), \quad \mathrm{SF}=(0.528), \quad \mathrm{CF}=(0.274) .
$$

The remaining pairwise comparison matrices can also be reckoned in the same way. Table 6 shows the complete FLPR matrix of sub-criteria with respect to vendor factors.
Notably, the matrix has entries that are not included in the interval $[0,1]$. Hence, the transforming function (Eq. 10) that preserves reciprocity and additive consistency is conducted. The results are exhibited in Table 7. The crisp values of fuzzy weights were computed thanks to Eq. (13) as follows.

$\mathrm{TS}=(0.256), \quad \mathrm{MP}=(0.325), \quad \mathrm{RD}=(0.185)$,
$\mathrm{GR}=(0.329)$

The decision matrix for FLPR of sub-criteria with respect to system factors is constituted similarly and shown in Table 8. Notably, there exist some entries that are not in interval $[0,1]$. Hence, the transforming function is again utilized. The results are exhibited in Table 9.

Table 6. Complete FLPR matrix of sub-criteria with respect to vendor factors

\begin{tabular}{lccccc}
\hline VF & TS & MP & RD & GR & Weight \\
\hline TS & $(0.50,0.50,0.50)$ & $(0.21,0.36,0.50)$ & $(0.36,0.64,0.86)$ & $(0.00,0.36,0.71)$ & $(0.10,0.23,0.45)$ \\
MP & $(0.50,0.64,0.79)$ & $(0.50,0.50,0.50)$ & $(0.64,0.79,0.86)$ & $(0.29,0.50,0.71)$ & $(0.18,0.30,0.51)$ \\
RD & $(0.14,0.36,0.64)$ & $(0.14,0.21,0.36)$ & $(0.50,0.50,0.50)$ & $(0.14,0.21,0.36)$ & $(0.09,0.16,0.33)$ \\
GR & $(0.29,0.64,1.00)$ & $(0.29,0.50,0.71)$ & $(0.64,0.79,0.86)$ & $(0.50,0.50,0.50)$ & $(0.16,0.30,0.55)$ \\
\hline
\end{tabular}

Table 7. Transformation matrix of sub-criteria with respect to vendor factors

\begin{tabular}{lccccc}
\hline VF & TS & MP & RD & GR & Weight \\
\hline TS & $(0.50,0.50,0.50)$ & $(0.21,0.36,0.50)$ & $(0.36,0.64,0.86)$ & $(0.00,0.36,0.71)$ & $(0.10,0.23,0.45)$ \\
MP & $(0.50,0.64,0.79)$ & $(0.50,0.50,0.50)$ & $(0.64,0.79,0.86)$ & $(0.29,0.50,0.71)$ & $(0.18,0.30,0.51)$ \\
RD & $(0.14,0.36,0.64)$ & $(0.14,0.21,0.36)$ & $(0.50,0.50,0.50)$ & $(0.14,0.21,0.36)$ & $(0.09,0.16,0.33)$ \\
GR & $(0.29,0.64,1.00)$ & $(0.29,0.50,0.71)$ & $(0.64,0.79,0.86)$ & $(0.50,0.50,0.50)$ & $(0.16,0.30,0.55)$ \\
\hline
\end{tabular}

Table 8. Complete FLPR matrix of sub-criteria with respect to system factors

\begin{tabular}{lccccc}
\hline SF & FU & RE & EC & EI & EF \\
\hline FU & $(0.5,0.5,0.5)$ & $(0.1,0.3,0.5)$ & $(0.3,0.7,1.0)$ & $(-0.1,0.5,1.0)$ & $(-0.1,0.7,1.4)$ \\
RE & $(0.5,0.7,0.9)$ & $(0.5,0.5,0.5)$ & $(0.7,0.9,1.0)$ & $(0.3,0.7,1.0)$ & $(0.3,0.9,1.4)$ \\
EC & $(0.0,0.3,0.7)$ & $(0.0,0.1,0.3)$ & $(0.5,0.5,0.5)$ & $(0.1,0.3,0.5)$ & $(0.3,0.5,0.9)$ \\
EI & $(0.0,0.5,1.1)$ & $(0.0,0.3,0.7)$ & $(0.5,0.7,0.9)$ & $(0.5,0.5,0.5)$ & $(0.5,0.7,0.9)$ \\
EF & $(-0.4,0.3,1.1)$ & $(-0.4,0.1,0.7)$ & $(0.1,0.5,0.7)$ & $(0.1,0.3,0.5)$ & $(0.5,0.5,0.5)$ \\
\hline
\end{tabular}


The crisp weights of each criterion extracted through defuzzification are as follows.

$\mathrm{FU}=(0.232), \quad \mathrm{RE}=(0.274), \quad \mathrm{EC}=(0.187)$,

$\mathrm{EI}=(0.233), \quad \mathrm{EF}=(0.188)$

Likewise, the complete pairwise comparison matrix of sub-criteria with respect to cost factors is illustrated in Table 10. There are entries not included in the interval $[0,1]$. Therefore, the transforming function is performed to normalizing the values. The converted values and the weight of each criterion are displayed in Table 11.
The crisp values of each fuzzy weight are reckoned by defuzzification as follows.

$$
\begin{aligned}
& \mathrm{OC}=(0.326), \mathrm{IC}=(0.282), \mathrm{MC}=(0.239), \\
& \mathrm{PR}=(0.199)
\end{aligned}
$$

Finally, the local and overall weights of all criteria were obtained and their normalized values are shown in Table 12. According to the Table 12, reliability (RE), ease of implementation (EI) and functionality (FU) emerged as the most important three criteria for the ERP selection process. Those criteria weights will be used in fuzzy TOPSIS procedure in an effort to specify the best ERP software alternative.

Table 9. Transformation matrix of sub-criteria with respect to system factors

\begin{tabular}{lcccccc}
\hline SF & FU & RE & EC & EI & EF & Weights \\
\hline FU & $(0.50,0.50,0.50)$ & $(0.28,0.39,0.50)$ & $(0.39,0.61,0.78)$ & $(0.17,0.50,0.78)$ & $(0.17,0.61,1.00)$ & $(0.09,0.23,0.42)$ \\
RE & $(0.50,0.61,0.72)$ & $(0.50,0.50,0.50)$ & $(0.61,0.72,0.78)$ & $(0.39,0.61,0.78)$ & $(0.39,0.72,1.00)$ & $(0.14,0.24,0.44)$ \\
EC & $(0.22,0.39,0.61)$ & $(0.22,0.28,0.39)$ & $(0.50,0.50,0.50)$ & $(0.28,0.39,0.50)$ & $(0.39,0.50,0.72)$ & $(0.10,0.18,0.32)$ \\
EI & $(0.22,0.50,0.83)$ & $(0.22,0.39,0.61)$ & $(0.50,0.61,0.72)$ & $(0.50,0.50,0.50)$ & $(0.50,0.61,0.72)$ & $(0.12,0.20,0.40)$ \\
EF & $(0.00,0.39,0.83)$ & $(0.00,0.28,0.61)$ & $(0.28,0.50,0.61)$ & $(0.28,0.39,0.50)$ & $(0.50,0.50,0.50)$ & $(0.06,0.15,0.37)$ \\
\hline
\end{tabular}

Table 10. Complete FLPR matrix of sub-criteria with respect to cost factors

\begin{tabular}{lcccc}
\hline CF & OC & IC & MC & PR \\
\hline OC & $(0.5,0.5,0.5)$ & $(0.5,0.7,0.9)$ & $(0.5,0.9,1.3)$ & $(0.5,1.1,1.7)$ \\
IC & $(0.1,0.3,0.5)$ & $(0.5,0.5,0.5)$ & $(0.5,0.7,0.9)$ & $(0.5,0.9,1.3)$ \\
MC & $(-0.3,0.1,0.5)$ & $(0.1,0.3,0.5)$ & $(0.5,0.5,0.5)$ & $(0.5,0.7,0.9)$ \\
PR & $(-0.7,-0.1,0.5)$ & $(-0.3,0.1,0.5)$ & $(0.1,0.3,0.5)$ & $(0.5,0.5,0.5)$ \\
\hline
\end{tabular}

Table 11. Transformation matrix of sub-criteria with respect to cost factors

\begin{tabular}{lccccc}
\hline CF & OC & IC & MC & PR & Weights \\
\hline OC & $(0.50,0.50,0.50)$ & $(0.50,0.58,0.67)$ & $(0.50,0.67,0.83)$ & $(0.50,0.75,1.00)$ & $(0.21,0.31,0.47)$ \\
IC & $(0.33,0.42,0.50)$ & $(0.50,0.50,0.50)$ & $(0.50,0.58,0.67)$ & $(0.50,0.67,0.83)$ & $(0.19,0.27,0.39)$ \\
MC & $(0.17,0.33,0.50)$ & $(0.33,0.42,0.50)$ & $(0.50,0.50,0.50)$ & $(0.50,0.58,0.67)$ & $(0.15,0.23,0.34)$ \\
PR & $(0.00,0.25,0.50)$ & $(0.17,0.33,0.50)$ & $(0.33,0.42,0.50)$ & $(0.50,0.50,0.50)$ & $(0.10,0.19,0.31)$ \\
\hline
\end{tabular}


Table 12. The overall weights of sub-criteria

\begin{tabular}{llll}
\hline $\begin{array}{l}\text { Criteria and Sub- } \\
\text { criteria }\end{array}$ & $\begin{array}{l}\text { Local } \\
\text { Weights }\end{array}$ & $\begin{array}{l}\text { Overall } \\
\text { Weights }\end{array}$ & Normalization \\
\hline Vendor Factors & 0.274 & & \\
TS & 0.256 & 0.070 & 0.060 \\
MP & 0.325 & 0.089 & 0.076 \\
RD & 0.185 & 0.051 & 0.043 \\
GR & 0.329 & 0.090 & 0.077 \\
System Factors & 0.528 & & \\
FU & 0.232 & 0.122 & 0.104 \\
RE & 0.274 & 0.145 & 0.124 \\
EC & 0.187 & 0.099 & 0.084 \\
EI & 0.233 & 0.123 & 0.105 \\
EF & 0.188 & 0.099 & 0.084 \\
Cost Factors & 0.274 & & \\
OC & 0.326 & 0.089 & 0.076 \\
IC & 0.282 & 0.077 & 0.066 \\
MC & 0.239 & 0.065 & 0.055 \\
PR & 0.199 & 0.054 & 0.046 \\
\hline
\end{tabular}

\subsubsection{Fuzzy TOPSIS Phase}

In the second phase of the study, the project team rated the alternative ERP systems with respect to the evaluation criteria by using the linguistic variables in Table 2. The decision matrix involving the ratings of alternatives is depicted in Table 13.

Having constructed the fuzzy decision matrix, we normalized it using Eqs. (17) and (18). Then, the weighted normalized fuzzy decision matrix depicted in Table 14 is calculated using Eq. (19).
Table 13. Fuzzy decision matrix

\begin{tabular}{lccc}
\hline & A & B & C \\
\hline TS & $(5,7,9)$ & $(5,7,9)$ & $(7,9,1)$ \\
MP & $(5,7,9)$ & $(9,1,1)$ & $(7,9,1)$ \\
RD & $(3,5,7)$ & $(7,9,1)$ & $(9,1,1)$ \\
GR & $(7,9,1)$ & $(7,9,1)$ & $(7,9,1)$ \\
FU & $(7,9,1)$ & $(5,7,9)$ & $(5,7,9)$ \\
RE & $(5,7,9)$ & $(7,9,1)$ & $(9,1,1)$ \\
EC & $(3,5,7)$ & $(5,7,9)$ & $(7,9,1)$ \\
EI & $(7,9,1)$ & $(7,9,1)$ & $(5,7,9)$ \\
EF & $(7,9,1)$ & $(7,9,1)$ & $(7,9,1)$ \\
OC & $(9,1,1)$ & $(7,9,1)$ & $(5,7,9)$ \\
IC & $(7,9,1)$ & $(5,7,9)$ & $(5,7,9)$ \\
MC & $(5,7,9)$ & $(3,5,7)$ & $(3,5)$, \\
PR & $(9,1,1)$ & $(5,7,9)$ & $(1,3,5)$ \\
\hline & & & \\
\end{tabular}

Herein, the weights used to compute weighted normalized fuzzy decision matrix are those elicited in the FLPR phase. In the next step, fuzzy positive ideal solution (FPIS, $A^{*}$ ) and fuzzy negative ideal solution (FNIS, $A^{-}$) are calculated through Eqs. (19) and (20). Since fuzzy preferences are normalized positive TFNs we specify the FPIS as $\tilde{v}_{j}^{*}=(1,1,1)$ and the FNIS as $\tilde{v}_{j}^{-}=(0,0,0)$. Afterwards, the distance of each alternative from FPIS and FNIS are computed by using Eqs. (21) and (22).

Table 14. The weighted normalized decision matrix

\begin{tabular}{llll}
\hline & $\mathrm{A}$ & $\mathrm{B}$ & \\
\hline TS & $(0.030,0.042,0.054)$ & $(0.030,0.042,0.054)$ & $(0.042,0.054,0.060)$ \\
MP & $(0.038,0.053,0.068)$ & $(0.068,0.076,0.076)$ & $(0.053,0.068,0.076)$ \\
RD & $(0.013,0.022,0.030)$ & $(0.030,0.039,0.043)$ & $(0.039,0.043,0.043)$ \\
GR & $(0.054,0.069,0.077)$ & $(0.054,0.069,0.077)$ & $(0.054,0.069,0.077)$ \\
FU & $(0.073,0.094,0.104)$ & $(0.052,0.073,0.094)$ & $(0.052,0.073,0.094)$ \\
RE & $(0.062,0.087,0.112)$ & $(0.087,0.112,0.124)$ & $(0.112,0.124,0.124)$ \\
EC & $(0.025,0.042,0.059)$ & $(0.042,0.059,0.076)$ & $(0.059,0.076,0.084)$ \\
EI & $(0.074,0.095,0.105)$ & $(0.074,0.095,0.105)$ & $(0.059,0.076,0.084)$ \\
EF & $(0.059,0.076,0.084)$ & $(0.059,0.076,0.084)$ & $(0.038,0.053,0.068)$ \\
OC & $(0.068,0.076,0.076)$ & $(0.053,0.068,0.076)$ & $(0.033,0.046,0.059)$ \\
IC & $(0.046,0.059,0.066)$ & $(0.033,0.046,0.059)$ & $(0.018,0.031,0.043)$ \\
MC & $(0.031,0.043,0.055)$ & $(0.018,0.0310 .043)$ & $(0.005,0.014,0.023)$ \\
PR & $(0.041,0.046,0.046)$ & $(0.023,0.032,0.041)$ & $(0.095)$ \\
\hline
\end{tabular}


Finally, the closeness coefficient of each alternative is calculated by means of Eq. (24) and preference orders are determined. The results are presented in Table 15. Consequently, alternative $\mathrm{C}$ is occurred as the best ERP software for the company and the full ranking of the alternatives is as $\mathrm{C}>\mathrm{B}>\mathrm{A}$.

Table 15. The results of fuzzy TOPSIS phase.

\begin{tabular}{ccccc}
\hline & $d_{i}^{*}$ & $d_{i}^{-}$ & $C C_{i}$ & Order \\
\hline $\mathrm{A}$ & 9.177 & 0.387 & 0.040 & 3 \\
$\mathrm{~B}$ & 9.177 & 0.396 & 0.041 & 2 \\
$\mathrm{C}$ & 9.170 & 0.402 & 0.042 & 1 \\
\hline
\end{tabular}

\section{Discussion}

In terms of pairwise comparison times, EAM requires $\left(C_{2}^{3}\right)+3 *\left(C_{2}^{4}\right)+3 *\left(C_{2}^{5}\right)+3 *\left(C_{2}^{4}\right)=69 \quad$ pairwise comparisons, for the three main criteria and 13 subcriteria in the FLPR phase of the study, which may cause an inconsistency problem. However, the FLPR method only required $(2+(3 * 3)+(3 * 4)+(3 * 3)=32$ pairwise comparisons and consistency is assured. That is to say, as the number of criteria and alternatives increase the number of pairwise comparison required by the FLPR method reduces with respect to EAM. Kahraman et al. ${ }^{48}$ proposed using fuzzy AHP for optimal location of an automobile manufacturer between three alternatives considering four criteria. If they used the FLPR approach was rather than fuzzy AHP, the number of pairwise comparisons would be reduced by $\left(\mathrm{C}_{2}^{4}-3\right)+4 *\left(\mathrm{C}_{2}^{3}-2\right)=7$ times while ensured consistency.

Önüt and Efendigil ${ }^{49}$ aimed to select the best ERP software system via fuzzy AHP where three alternatives were evaluated with respect to three main and ten subcriteria. The fuzzy AHP application entailed 53 pairwise comparisons whereas the FLPR method could require $\left(C_{2}^{3}-2\right)+\left(C_{2}^{6}-5\right)+\left(C_{2}^{2}-1\right)+\left(C_{2}^{2}-1\right)+10 *\left(C_{2}^{3}-2\right)=21$ times less computation. In addition, the fuzzy AHP assigned a zero value of weight to one main criterion and three sub-criteria, which is irrational. Hence, those criteria were excluded from the decision analysis. However, we can circumvent such unreasonable conditions by using the proposed FLPR approach.

\section{Conclusions}

Recently, Zeydan et al. ${ }^{50}$ conducted a large literature survey claiming that hybrid MCDM techniques such as AHP/TOPSIS, ANP/ fuzzy TOPSIS and TOPSIS/DEA etc. do not have adequate variety of applications in the literature. This research has attempted to propose a fresh decision support tool for selecting the best ERP software/vendor alternative by integrating Delphi technique, FLPR and Fuzzy TOPSIS methods. Using fuzzy theory for ERP system selection can reduce ambiguities and vagueness inherent in the decisionmaking process. In order to show the viability of the proposed evaluation framework, a real case application in a Turkish wholesale food company has been conducted. The combined evaluation model is designed to provide practitioners with a fuzzy point of view to simplify the decision-making phase with imprecise or vague environments by using linguistic preferences.

Fuzzy TOPSIS is a useful tool to handle with the ratings of both quantitative as well as qualitative criteria/alternatives and selects the appropriate ERP alternative effectively. Furthermore, it does not impose any restriction on the number of alternatives or criteria used in the selection process. The elicitation of the relative weights of the evaluation criteria/attributes is a crucial step in fuzzy TOPSIS in that it directly affects the selection of the optimum alternative. In the MCDM literature, it is seen that the AHP and ANP methods and their fuzzy models are the foremost approaches used together with TOPSIS method to determine attributes' weights. For instance, Torfi et al. ${ }^{51}$ and Taylan et al. ${ }^{52}$ exploited fuzzy AHP while Kabak et al. ${ }^{53}$ preferred fuzzy ANP so as to specify the weights of the criteria to be used in the TOPSIS phase of their case studies. However, inconsistency increases as hierarchies of criteria or alternatives increase in both AHP and ANP as previously mentioned. Since the lack of consistency in decision making can result in inconsistent conclusion, this study adopted FLPR for deriving consistent weights of evaluation criteria based on pairwise comparisons. Moreover, in EAM, the weights of some decision criteria would be zero, which means it has no effect in the decision process. Using FLPR can avoid such unreasonable conditions. Applying the proposed hybrid method it is aimed to exploit the advantageous characteristics of both methods. 
The presented methodology would be useful for the industry as it incorporates the full support of the management to utilize their experiences concerning the business processes of the firm and thus eliminate the biases in the selection procedure of the appropriate ERP system. In addition, the proposed hybrid model provides the opportunity to the ERP project team to break down such a complicated selection problem into smaller components. Hence, the team can comprehend the relationships between various attributes and assign easily their judgments to the hierarchical structure. Perfect consistency is difficult to secure in practice, particularly when gauging preferences on a set with many alternatives. By using the proposed methodology, the consistency of the fuzzy preference relations provided by decision makers is improved, such that inconsistent solutions in decision making processes are avoided. Besides, the developed framework is flexible enough to incorporate extra attributes or decision makers in the process. Furthermore, it can hasten the reaching of consensus among multiple decision makers, which will reduce resistance. It is also worth noting that the decision making approach presented in this study is not limited to ERP system selection. The FLPR provides greater flexibility for solving MCDM problems with preference information concerning alternatives and/or attributes. Future research will focus on realworld applications of the proposed framework to cope with other MCDM problems. Despite aforementioned merits, this study has some limitations, as well. The introduced framework does not allow taking account for the interdependencies between decision criteria, which can be considered as a drawback when applied to the best ERP software selection problem. Ignoring such interactions may lead us to get a different final ranking. A future study can be extended to cover interactions and inner or outer dependencies among criteria or alternatives with the fuzzy ANP to verify the findings of the present study. Furthermore, the ranking of the ERP alternatives could be employed with the other crisp or fuzzy versions of MCDM methods such as PROMETHEE, ELECTRE and VIKOR etc. and the results can be compared.

\section{Acknowledgements}

The author would like to thank anonymous reviewers for their valuable comments and suggestions to improve the quality of this paper.

\section{References}

1. H.R.Yazgan HR, S. Boran, K. Göztepe, An ERP software selection process with using artificial neural network based on analytic network process approach, Expert Systems with Applications 36 (2009) 9214-9222.

2. C.C Wei, C.F. Chien, M.J Wang, An AHP-based approach to ERP system selection, International Journal of Production Economics, 96 (2005) 47-62.

3. U.Cebeci, Fuzzy AHP-based decision support system for selecting ERP Systems in textile industry by using balanced scorecard, Expert Systems with Applications $\mathbf{3 6}$ (2009) 8900-8909.

4. S. Önüt, T. Efendigil, Theorical model design for ERP software selection process under the constraints of cost and quality: A fuzzy approach, Journal of Intelligent \& Fuzzy Systems 21 (2010) 365-378.

5. Y. Yusuf, A. Gunasekaran, Abthorpe MS. Enterprise information systems project implementation: A case study of ERP in Rolls-Royce, International Journal of Production Economics 87 (2004) 251-266.

6. J. Ram, M.L. Wu, R. Tagg, Competitive advantage from ERP projects: Examining the role of key implementation drivers, Int. J. of Project Management 32 (2004) 663675.

7. E. Karsak, C.O. Özoğul, An integrated decision making approach for ERP system selection, Expert Systems with Applications 36 (2009): 660-667.

8. T.L. Saaty, The Analytic Hierarchy Process. McGrawHill, New York, 1980.

9. Y.H. Chen, T.C. Wang, C.Y. Wu, Multi-criteria decision making with fuzzy linguistic preference relations, Appl. Mathematic Modelling 35 (2001) 1322-1330.

10. P.J.M. Laarhoven, W. Pedrycz, A fuzzy extension of Saaty's priority theory, Fuzzy Sets and Systems 11 (1983) 229-241.

11. J.J. Buckley, Fuzzy hierarchical analysis, Fuzzy Sets and Systems 17 (1985) 233-247.

12. D.Y. Chang, Applications of the extent analysis method on fuzzy AHP, European Journal Operations Research 95 (1996) 649-655.

13. E. Herrera-Viedma, F. Herrera, F. Chiclana, M. Luque, Some issues on consistency of fuzzy preference relations, European Journal Operations Research 154 (2004): 98109. 
14. Wang TC, Chen YH. Fuzzy multi-criteria selection among transportation companies with fuzzy linguistic preference relations Expert Systems with Applications $\mathbf{3 8}$ (2011):11884-11890.

15. T.C. Wang, Y.H. Chen, A new method on decisionmaking using fuzzy linguistic assessment variables and fuzzy preference relations, In Paper presented at the 9th world multi-conference on systemic, cybernetics and informatics (WMSCI 2005), Orlando, Florida, USA, 2005.

16. T.C. Wang, Y.H. Chen, Applying fuzzy linguistic preference relations to the improvement consistency of fuzzy AHP, Information Sciences 178 (2008) 3755-3765.

17. T.C. Wang, Y.H. Chen, Incomplete fuzzy linguistic preference relations under uncertain environment, Information Fusion 11 (2010) 201-207.

18. Y.H. Chen, On consistency of fuzzy analytic hierarchy process. Unpublished doctoral dissertation, I-Shou University, Kaohsiung, Taiwan. 2008.

19. C.L. Hwang, K. Yoon, Multiple attribute decision making: methods and applications. In Lecture notes in economics and mathematical systems, Springer, New York, 1981.

20. C.T. Chen, Extensions of the TOPSIS for group decisionmaking under fuzzy environment, Fuzzy Sets and Systems 114 (2000) 1-9.

21. H.C Lucas, J.R. Moore, A multiple-criterion scoring approach to information system project selection, Informs 14 (1976): 1-12.

22. C.A. Ptak, ERP Tools, Techniques, and Applications for Integrating the Supply Chain. St. Lucie Press, New York, 2000.

23. M.D.J. Buss, How to rank computer projects, Harvard Business Review 61 (1983) 118-125.

24. R. Santhanam, G.J. Kyparisis, A multiple criteria decision model for information system project selection, Computers \& Operations Research 22 (1995) 807-818.

25. J.W. Lee, S.H. Kim, Using analytic network process and goal programming for interdependent information system project selection, Computers \& Operations Research 27 (2000) 367-382.

26. V. Lall, S. Teyarachakul, Enterprise resource planning (ERP) system selection: A data envelopment analysis (DEA) approach, Journal of Computer Information Systems 47 (2006) 123-7.

27. M.A, Badri, D. Davis, D.A. Davis, A comprehensive 0-1 goal programming model for project selection, International. Journal of Project Management 19 (2001) 243-252.

28. A. Teltumbde, A framework for evaluating ERP projects, International Journal of Production Research 38 (2000) $4507-4520$.
29. C.C. Wei, M.J. Wang, A comprehensive framework for selecting an ERP system, International Journal of Project Management 22 (2004) 161-169.

30. N. Karaaslan, E. Gündoğar. An application for modular capability-based ERP software selection using AHP method, International. Journal of Advanced Manufacturing Technologies 42 (2009) 1025-1033.

31. C.T. Lin, C.B. Chen, Y.C. Ting, An ERP model for supplier selection in electronics industry, Expert Systems with Applications 38 (2011) 1760-1765.

32. T. Gürbüz, S.E. Alptekin, G. Alptekin Iş̧1klar, A hybrid MCDM methodology for ERP selection problem with interacting criteria, Decision Support Systems 54 (2012) 206-214.

33. G. Zhang, J. Ma, J. Lu, Emergency management evaluation by a fuzzy multi-criteria group decision support system, Stochastic Environmental Research and Risk Assessment 23 (2009), 517-527

34. J. Lu, Y. Zhu, X. Zeng, L. Koehl, J. Ma, G. Zhang, A linguistic multi-criteria group decision support system for fabric hand evaluation Fuzzy Optimization and Decision Making 8 (2009), 395-413.

35. L.A. Zadeh, Fuzzy sets, Information Control 8 (1965) 338-353.

36. G. Bojadziev, M. Bojadziev, Fuzzy Logic for Business, Finance and Management, 2nd edition World Scientific Publishing, London, 2007.

37. F. Chiclana, Herrera, E. Herrera-Viedma, Integrating three representation models in fuzzy multipurpose decision making based on fuzzy preference relations, Fuzzy Sets and Systems 97 ( 1998) 33-48.

38. J. Fodor, M. Roubens, Fuzzy preference modelling and multicriteria decision support, Kluwer, Dordrecht, 1994.

39. T. Tanino, Fuzzy preference relations in group decision making. In: Kacprzyk J, Roubens M, editors. Nonconventional preference relations in decision making, Berlin: Springer; 1988, pp. 54-71.

40. M. Fedrizzi, On a consensus measure in a group MCDM problem. In J. Kacprzyk \& M. Fedrizzi (Eds.), Multi person decision making models using Fuzzy sets and possibility theory, New York: Kluwer Academic, 1990, pp.231-41.

41. E. Triantaphyllou, C.T. Lin, Development and evaluation of five fuzzy multi-attribute decision-making methods, International. Journal of Approximate Reasoning 14 (1996) 281-310.

42. T.C. Chu, Y.C. Lin, A fuzzy TOPSIS method for robot selection, International. Journal of Advanced Manufacturing Technologies 21 (2003) 284-290.

43. G.R. Jahanshahloo, F. Hosseinzadeh Lotfi, M. Izadikhah, Extension of the TOPSIS method for decision-making problems with fuzzy data, Applied Mathematics and Computation 181 (2006) 1544-1551. 
44. R.A. Krohling, V.C. Campanharo, Fuzzy TOPSIS for Group Decision Making: A Case Study for Accidents with Oil Spill in the Sea Expert Systems with Applications 38 (2011) 4190-4197.

45. T. Paksoy, N.Y. Pehlivan, C. Kahraman, Organizational strategy development in distribution channel management using fuzzy AHP and hierarchical fuzzy TOPSIS, Expert Systems with Applications 39 (2012) 2822-2841.

46. C.T. Chen, C.T. Lin, S.F. Huang, A fuzzy approach for supplier evaluation and selection in supply chain management, International Journal of Production Economics 102 (2006) 289-301.

47. G. Rowe, G. Wright, The Delphi technique as a forecasting tool: issues and Analysis. International Journal of Forecasting 1 (1999) 353-375.

48. C. Kahraman, D. Ruan, İ. Doğan, Fuzzy group decisionmaking for facility location selection, Information Sciences 157 (2003) 135-153.
49. S. Önüt, T. Efendigil, A theorical model design for ERP software selection process under the constraints of cost and quality: A fuzzy approach, Journal of Intelligent \& Fuzzy Systems 21 (2010) 365-378.

50. M. Zeydan, C. Colpan, C., Cobanoğlu, A combined methodology for supplier selection and performance evaluation, Expert Systems with Applications, 38 (2011) 2741-2751.

51. F. Torfi, R. Z. Farahani, S. Rezapour, Fuzzy AHP to determine the relative weights of evaluation criteria and Fuzzy TOPSIS to rank the alternatives, Applied Soft Computing 10 (2010) 520-528.

52. O. Taylan, A.O. Bafail, R.M.S. Abdulaal, M.R. Kabli, Construction projects selection and risk assessment by fuzzy AHP and fuzzy TOPSIS methodologies, Applied Soft Computing 17 (2014) 105-116.

53. M. Kabak, S. Burmaoğlu, Y. Kazançoğlu, A fuzzy hybrid MCDM approach for professional selection, Expert Systems with Applications, 39 (2012) 3516-3525. 